\title{
EL ÁNGULO MUERTO DE HART. LA PIEZA QUE FALTA EN THE CONCEPT OF LAW
}

\author{
Bruno Celano \\ Università degli Studi di Palermo
}

RESUMEN. Al inicio de El Concepto de Derecho, HART sugiere una asimilación errónea entre conducta que es «no opcional» y conducta que es "obligatoria» (i. e. conducta que o es forzada o sometida a una obligación). Esta asimilación vicia el argumento del libro en su conjunto, llevándole a HART a descuidar las diferentes vías en las que el derecho monitorea, corrobora o constituye relaciones de poder. Es cierto y evidente que en The Concept of Law la atención se dedica a los poderes normativos, legales. Sin embargo, el poder social bruto, y las relaciones del derecho con él -el papel del derecho como una pieza en la maquinaria de los poderes sociales-están ampliamente ignorados. Éste es, de alguna manera, el ángulo muerto de HART.

Enuncio algunas de las vías, aparte de la coerción u obligación, en las que el derecho puede servir, corroborar o ser un instrumento de poder social. También muestro que el tratamiento de HART de las relaciones entre el derecho y el poder político, en The Concept of Law, es insatisfactorio. Tanto su reconstrucción de la surgimiento ideal de un sistema jurídico desarrollado y el argumento que sustenta «el contenido mínimo del derecho natural» presuponen la idea de un grupo social en el que no subsisten relaciones de poder.

Palabras clave: H. L. A. HART, poder, filosofía del derecho analítica, filosofía jurídica y política, Estado de derecho, normas que confieren poderes, derecho e ideología.

\section{Hart's blind spot. The missing piece in The Concept of Law}

ABSTRACT. At the beginning of The Concept of Law HART suggests a mistaken assimilation between conduct that is «non-optional» and conduct that is «obligatory» (i. e. conduct that is either coerced or subject to an obligation). This suggested assimilation vitiates the argument of the whole book, leading HART to neglect the different ways in which the law typically tracks, corroborates or constitutes power relations. It is true that, famously, attention is paid, in The Concept of Law, to normative, legal powers. Brute social power, and law's relation to it -the role of law as a cog in the workings of social powers-, however, are largely overlooked. This is, in a way, HART's blind spot. I list some of the ways, other from coercion or obligation, in which the law may happen to serve, corroborate or be an instrument of social power. I also show that HART's treatment of the relations between the law and political power, in The Concept of Law, is unsatisfactory. Both his reconstruction of the ideal genesis of a developed legal system and the argument supporting a «minimal content of natural law» presuppose the idea of a social group in which no power relationships subsist.

Keywords: H. L. A. HART, power, analytical jurisprudence, legal and political philosophy, rule of law, power-conferring rules, law and ideology.

* Fecha de recepción: 5 de septiembre de 2014. Fecha de aceptación: 21 de octubre de 2014.

Una versión inicial del presente trabajo se presentó en el XVIII Congreso Hispano-Italiano-Francés de Teoría del Derecho celebrado en la Universidad Carlos III de Madrid bajo la organización del Grupo de Investigación sobre el Derecho y la Justicia (GIDYJ). 


\section{EL PUNTO CIEGO}

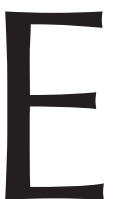

n la introducción a The Concept of Law (en adelante, CL) HART observa: «The most prominent general feature of law at all times and places is that its existence means that certain kinds of human conduct are no longer optional, but in some sense obligatory» («la característica general más destacada del derecho, en todo tiempo y lugar, es que su existencia significa que ciertos tipos de conducta humana no son ya optativos sino obligatorios, en algún sentido», CL: 6; versión española —en adelante CD—: 7; cfr. también CL: 80, CD: 102: «Where there is law, there buman conduct is made in some sense non-optional or obligatory») ${ }^{1}$.

Esta observación parece inocua pero tiene trampa. La observación sugiere una identificación: la identificación entre ser una línea de conducta «no opcional» y ser una «obligatoria» y esta identificación es falaz. HART aclara explícitamente que la característica en cuestión - el ser un cierto tipo de comportamiento no opcional y obligatorio- no es «simple». Escribe: «es posible distinguir dentro la esfera de la conducta no optativa u obligatoria, diversas formas» («within the sphere of non-optional or obligatory conduct we can distinguish different forms», CL: 6; CD: 8). Hay así varios sentidos o modos en los que un cierto comportamiento puede decirse no opcional (o no más opcional) u obligatorio. La distinción que aquí tiene HART en mente y que analiza es, como veremos a continuación, la que media entre el ser obligado y tener una obligación. Pero, repito, el problema es — a contracorriente respecto de esta distinción- la identificación que HART sugiere y parece sobreentender (pero no afirma ni, menos aún, argumenta) entre lo no (más) opcional y lo obligatorio.

«The simplest sense in which conduct is no longer optional», prosigue HART «is when one man is forced to do what another tells him; no because be is physically compelled in the sense that his body is pushed or pulled about, but because the other threatens bim with unpleasant consequences if he refuses» («El sentido primero, y el más simple, en que la conducta no es ya optativa, se presenta cuando un hombre se ve forzado a hacer lo que otro le dice, no porque sea compelido físicamente en el sentido de que se actúa sobre su cuerpo, sino porque el otro lo amenaza con consecuencias desagradables si se rehúsa a hacer lo que éste quiere», CL: 6; CD: 8). Este tipo de situación es ejemplificado por el caso de un asaltante que amenaza a su víctima con un cuchillo ordenándole que le entregue la bolsa. En un caso de este tipo, señala HART, aquél al que se le dice hacer una cierta cosa si efectivamente la hace (si el viajero entrega la bolsa al bandido) se dirá que él «ha estado obligado» a actuar así («be was obliged to do so») (CL: 6; CD: 8).

El segundo sentido, «menos simple», en que la conducta humana es «no-opcional, obligatoria» («a second way in which conduct may be not optional but obligatory», «una segunda manera en que la conducta puede ser no optativa sino obligatoria» CL: 7; CD: 8-9) que HART toma en consideración en CL es que un cierto comportamiento sea moralmente obligatorio (HART buscará así extraer y definir un tercer sentido, el de la obligación jurídica). De aquí la impostación de fondo del libro: «Promover la

${ }^{1}$ Éste, dice HART, es el «punto de partida» tanto de la teoría de AUSTIN como de su propia teoría (CL: 80; 
comprensión de derecho, coerción y moralidad como fenómenos sociales diferentes pero conexos» (CL: V, CL: X): el «fin (purpose)» del libro es «[to provide] a better understanding of the resemblances and differences between law, coercion, and morality, as types of social phenomena» («una mejor comprensión de las semejanzas y de las diferencias entre derecho, coerción y moralidad como tipos de fenómenos sociales», CL: 17; CD: 21. Cfr. también EJP: 91; Comment: 38; PS: 240)². De aquí que su problema fundamental sea determinar cuáles son las relaciones que se establecen entre el derecho, por un lado, y la coerción y la moral, por el otro 3 .

En el fondo, lo que queda es la aparentemente implicada pero no tematizada ni puesta en cuestión, identificación entre lo no-opcional y lo obligatorio ${ }^{4}$. Ahora bien: «obligatorio» no es la negación de «opcional». Más bien: (1) hay modos de expresar la conducta no-opcional que no consisten ni en la manipulación física (compulsión, apremio) de la víctima ${ }^{5}$, ni en hacer la conducta obligatoria (coerción o imposición de obligaciones). Hay otros modos entre los cuales hay algunos que son formas de ejercicio de poder; de poder social. (2) El derecho refleja, sanciona, corrobora, constituye algunos de ellos - algunas de las relaciones de poder conforman uno u otro de estos modos. (3) El CL, en razón del deslizamiento conceptual que he puesto a la luz y de la impostación problemática de fondo que hemos visto («promover la comprensión del derecho, coerción y moralidad como tres fenómenos sociales diferentes pero conectados» CL: V; CD: XI) se induce que, en los límites, queda ciego a esta dimensión del derecho; queda ciego respecto a las muchas las relaciones posibles entre derecho y poder.

En otros términos, la cuestión de las relaciones entre derecho y poder no se agota en la cuestión de la relación entre derecho y coerción. Pero HART, en el CL, colige solamente este último aspecto de las relaciones entre derecho y poder. Éste es el punto ciego de CL.

${ }^{2}$ HART añade que (CL: 17; CD: 22) la indicación de un segundo objetivo: «[to provide] an improved analysis of the distinctive structure of municipal legal systems». Pero no hay duda de que el problema al que se dirige la construcción del CL y que gobierna el desarrollo de la investigación es el indicado en el texto: «Delimitar el campo del derecho común tertium quid entre coerción (estar obligado) y moralidad (obligación moral). La estructura del libro es transparente, los Capítulos II-IV están dedicados a los argumentos que se oponen a la reducción del derecho a la coerción; los Capítulos VIII y IX que se oponen a la tesis de que el derecho sea una rama de la moralidad. La caracterización, en positivo, del derecho como unión de reglas primarias y secundarias (Caps. V y VI) adquiere su verdadero significado en el rechazo de estas dos hipótesis reduccionistas».

${ }^{3}$ HART menciona que (CL: 13; CD: 16) un tercer recurrent issue «what are rules and to what extent is law an affair of rules?» («¿Qué son las reglas, y en qué medida el derecho es una cuestión de reglas?»). Como es sabido, el análisis de la noción de existencia de una regla social y del sentido en que el derecho es una práctica gobernada por reglas, es uno de los temas centrales del libro. Aunque bajo este aspecto, sin embargo, la jurisprudence hartiana gira en torno al doble problema de la delimitación del derecho respecto de la coerción, de un lado; y respecto a la moral, del otro indicado en el texto ( $v i d$. la nota precedente). Por un lado, el ser una práctica rule governed es la que diferencia al derecho de la coerción [en particular, respecto a un conjunto de órdenes sostenidas por amenazas formuladas por un individuo o un grupo ante las cuales se da un hábito general de obediencia (CL, Cap. IV). Por otro lado, obligación jurídica y obligación moral son reconducibles a un conjunto diferente de reglas de diferente tipo (CL: 163-176; CD: 208-215 y Cap. IX)].

${ }^{4} \mathrm{La}$ redacción de HART «[T] he sphere of non-optional conduct» es una expresión ambigua. Si entre los términos «non-optional» y «obligatory» hubiese una coma, la identificación sería explícita, pero si la alternativa - si lo obligatorio es una forma particular de lo no opcional, o sea, lo mismo que lo no opcional, permanece la duda-. Otras formulaciones son ambiguas: «A second way in which conduct may be not optional but obligatory» (CL: 7; CD: 8-9, la cursiva es mía); «non-optional or obligatory» (CL: 80; CD: 102).

5 Se trata de una posibilidad que, como hemos visto, HART menciona pero que, en el CL, no estudia. El hecho de que la mencione implica que HART, en caso de preguntárselo, ciertamente no sostendría que «obligatorio» es la negación de «opcional» pero la cuestión permanece abierta. 


\section{EL DERECHO COMO PODER: ALGUNAS VARIACIONES}

En este punto, surgirán espontáneamente dos objeciones:

1) ¿Quizá HART no ha «descubierto» la clase de normas que confieren poderes? ¿Cómo se puede sostener que la fallida consideración de la relación entre derecho y poder es el punto ciego de CL?

2) Puede ocurrir, advierte HART, que un sistema jurídico reserve su protección, y los otros beneficios que derivan de su existencia, a una clase particular de individuos a quienes se aplican las reglas. ¿No es ésta una forma - la forma - en que puede ocurrir que el derecho refleje y concurra a constituir relaciones de poder social?

Un tercer punto, así, requiere clarificación. La segunda premisa de mi argumento (supra, 1, penúltimo párrafo) es que el derecho refleja, sanciona, corrobora, constituye algunas formas, diferentes de la coerción o de la imposición de obligaciones (y de la compulsión física en el sentido de que se actúa sobre su cuerpo) en las que el comportamiento humano puede ser calificado como no-opcional y entre las cuales algunas son formas de ejercicio del poder; constituyen formas de poder social. ¿Qué significa esto? ¿Qué formas o modos? ¿Qué relaciones de poder?

Considero el primer y el tercer punto en este parágrafo (respectivamente, puntos 2.1 y 2.2). La segunda objeción será discutida en el próximo parágrafo (infra 3).

Una ulterior objeción podría ser ésta: HART está, en general, muy atento a la especificidad, sea en ámbito moral sea jurídico, de la noción de obligación en sentido estricto (cfr. paradigmáticamente, HART, 1955: 79-80), donde lamenta el uso indiferenciado de «obligación» para indicar, genéricamente, cualquier cosa que está prescrita o que se debe (ought) hacer ( $c f r$ también HART, 1958: 83, 100). Por ello, así prosigue la objeción, no se le puede imputar un uso genérico e indiferenciado de obligation (como uno que es distinto de la coerción y de la compulsión física sobre el cuerpo de la víctima) como negación de «opcional».

Esto es cierto. Cuando HART discute específicamente la noción de obligación, es, como siempre, muy sensible a la necesidad, o simplemente a la oportunidad, de trazar distinciones. Pero ya he aclarado que el deslizamiento conceptual subrayado en el parágrafo precedente no se convierte en la atribución a HART de tesis explícitas: si se las hubiese planteado, ciertamente, él las habría rechazado. Se trata, más bien, de un tipo trompe-l'oeil, un deslizamiento inadvertido que condiciona, viciándolo, el completo planteamiento del libro y la formulación de su problema de fondo. Y, como ahora veremos, un escrutinio de los lugares en que HART discute específicamente la noción de obligation no induce a abandonar esta idea.

En un primer momento (HART, 1955) ${ }^{6}$, la noción de obligation tiene su lugar, propiamente, como correlativo de (claim-)right, en el cuadro de una choice theory de los derechos subjetivos ( $c f r$. para una comparación detallada CELANO, 2002: 19-32). Después (HART, 1958: 100 y ss.), la noción de obligation —o indiferentemente, duty que

\footnotetext{
${ }^{6}$ Es cierto que HART se ha retractado posteriormente de este escrito ( $c f r$. EJP: 17) pero por razones que no tienen nada que ver con esta elección terminológica [que aflorará también en algunos de los escritos posteriores dedicados a la elaboración y defensa de la choice theory de los derechos (EB: 184)].
} 
HART trata aquí los dos términos como sinónimos (ibid., 84) — es definida sobre la base de tres elementos: la dependencia de reglas sociales, la independencia del contenido (lo que significa que la obligation de hacer A podría, dentro de ciertos límites, fundar la obligation de no hacer A) y la perspectiva de la coerción ${ }^{7}$. Incluso, en el CL in fine (83-85; CD: 104-106), delimita, en el campo de los conceptos normativos, un papel específico para la noción de obligation. No todas las reglas sociales que dicen que se deben (ought) cumplir de un determinado modo imponen una obligation o duty (también aquí obligation es utilizado como sinónimo de duty; cfr. también EJP: 92), sino sólo aquellas que satisfacen tres condiciones: son sostenidas por una seria presión social; son concebidas por parte de los miembros del grupo como necesarias a los fines del mantenimiento de la vida asociada, o de algún aspecto de esta última considerado particularmente importante; imponen a aquellos que están sometidos a las mismas, sacrificios y renuncias ${ }^{8}$.

Estas precisiones y delimitaciones conceptuales, sin embargo, complican el cuadro pero no cambian la sustancia del problema. Complican el cuadro: las definiciones de obligation que HART introduce en oposición al uso genérico e indiferenciado del término como sinónimo de ought — respectivamente (1) como correlato de claim-right (HART, 1955), y (2) en relación con la noción de regla social (en HART, 1958 y, después en CL) no coinciden. Paradójicamente, la noción específica de obligation (obligation en sentido estricto) no es una sino (al menos) dos ${ }^{9}$ (la primera —obligation como correlativo de claim-right- es un caso particular de la segunda; CL: 85; CD: 104). Pero todo esto no cambia la sustancia del problema por dos razones: En primer lugar, en CL, no obstante la explícita precisión apenas mencionada, HART utiliza igualmente «obligation» cuando discute de reglas jurídicas en el sentido genérico de «ought», o sea, en el sentido de «reglas que prescriben que se debe comportar en un cierto modo». En segundo lugar, y lo más importante, ya sea obligation en sentido estricto, o de ought, tenemos todavía que ocuparnos, en contextos jurídicos, de la cualificación deóntica de los comportamientos sobre la base de normas de conducta. Y esto es suficiente para que se produzca el deslizamiento, el efecto de trompe-l'oeil, que he indicado. Después de todo, el mismo HarT en un pasaje crucial (en el contexto del análisis de la noción de legal obligation contenida en EB, Capítulo VI, por cuanto sé, la última palabra de HART sobre el tema) refiere a un elemento común el significado de duty (que también aquí trata como sinónimo de obligation) y el de ought en contextos jurídicos: la idea que ciertas acciones sean debidas (due from or owed by) por parte de ciertos individuos «en el sentido de que ellas pueden ser correctamente solicitadas o exigidas de ellos» $(160 ; c f r \text {. también EJP, } 93)^{10}$.

\footnotetext{
7 Caso paradigmático: las obligaciones derivadas de la promesa. Sin embargo, HART toma en consideración también las obligations o duties asociadas a los roles sociales en el caso en que las dos primeras características se presenten de manera diferente. La definición de la noción de obligation contenida en estas páginas es el paso intermedio entre la adoptada en HART, 1955 y la contenida en el CL (que se ve en el texto).

8 Además se ve el juego de los términos «duty» y «obligation» en EB: 182-188. Aquí, HarT reserva de hecho el término para la noción correlativa a la de claim-right, tratando la obligation como una especie de duty. Pero se trata de un expediente retórico y no de una elección terminológica y conceptual definida y explícita. Por el contrario, al inicio de este pasaje - EB: 182- HART escribe como si hubiese introducido una distinción entre dos tipos de obligation.

9 «Al menos» dos porque, como se ha subrayado, el tratamiento contenido en HART, 1958, indica un paso intermedio.

10 De la misma manera, HART es perfectamente consciente de la complejidad de las nociones de «voluntario» e «involuntario» (cfr. paradigmáticamente HART, 1949: 180, 190-193) (es cierto que también este escrito
} 


\subsection{Poderes normativos}

Según HART, uno de los rasgos distintivos de un sistema jurídico desarrollado es la presencia de normas que confieren poderes. En antítesis respecto a una representación pacatamente imperativista del derecho, en CL no se hace más que hablar de poderes, públicos o privados. ¿Cómo se puede sostener que la relación entre derecho y poder es el punto ciego de CL?

Simple: los poderes de los que se discute ampliamente en CL son poderes normativos (jurídicos): poderes, instituidos por normas (jurídicas), de producción y aplicación de normas (jurídicas). Son, así, poderes mediante los que el derecho regula su propia producción o aplicación: legal powers (CL: 32; CD: 45; cfr. para una mirada retrospectiva EB: 194-96). Pero ésta es sólo una de las caras del poder, una entre las muchas formas de poder social ${ }^{11}$. Hay muchas otras formas de poder social, en particular, formas de poder físico o psicológico no consistente en el ejercicio de poderes normativos ${ }^{12}$.

La única forma de poder (aparte de los poderes normativos) que, en CL, el derecho señala es el «poder coercitivo (coercive power)», consistente en la capacidad de obtener la ejecución o la omisión de ciertas acciones mediante la emisión de amenazas creíbles de sanciones punitivas en caso de incumplimiento. Y ésta es una limitación grave $^{13}$.

ha sido posteriormente revisado por HART pero, también en este caso, por razones que no tienen nada que ver con este punto).

11 En EB: 196-200, HART discute, siguiendo a BENTHAM, los poderes normativos (jurídicos) en un sentido posterior respecto a aquél —único presente en CL- introducido en el texto: los poderes conferidos por normas permisivas, o derivadas de la ausencia de normas, que llevan a término actos de manipulación de cosas o personas. A nuestros fines, esto no cambia las cosas porque se trata en todo caso de la cualificación deóntica — como (jurídicamente) permitida - de ciertas conductas (cfr. también ibid., 210). Cuando (ibid., 217-218), a los fines de aclarar la naturaleza de los poderes jurídicos (en particular, los poderes de los particulares), discute de «poderes informales no jurídicos» HART se refiere, en todo caso, a los «poderes normativos», producidos por «efectos normativos» (caso paradigmático: la promesa).

12 Se objetará: éstas son cuestiones objeto de la sociología del derecho y no de la filosofía del derecho o jurisprudence. Pero lo que está en discusión aquí es, precisamente, la naturaleza - los límites- de la jurisprudence. En general, creo que es bien escaso el beneficio a obtener de levantar empalizadas disciplinarias en este nivel de abstracción (no se está discutiendo, obviamente, de estudios empíricos de campo). Ciertamente, está totalmente fuera de lugar si miramos la perspectiva adoptada por HART en CL. El libro contiene muchas investigaciones y tesis de carácter sociológico — tres ejemplos: la investigación sobre las condiciones de existencia de una regla social; la tesis según la cual las reglas que imponen obligaciones y las reglas que confieren poderes se diferencian por su «función social»; la idea de que el fundamento de un sistema jurídico sea una regla de reconocimiento (como la entiende HART) — y es calificado por el propio HART como (y efectivamente es) «un ensayo de sociología descriptiva» [CL: V; CD: XI; el presenta «a theroy of what law is» que «seeks to give an explanatory and clarifying account of law as a complex social and political institution with a rule-governed (and in this sense "normative") aspect», PS: 239; cfr. también ibid., 246; Comment: 36-38]. Sobre la continuidad, según HART, entre investigaciones socio-jurídicas y filosofía del derecho (o, indiferenciadamente, jurisprudence) $c f r$. EJP: 88.

13 HarT entiende por «coerción» la amenaza de una «reacción hostil» (CL: 10; CD: 12-13) en el caso de incumplimiento ( $c f r$ también HART, 1955: 77, n. 2). Otros modos de ejercicio de la fuerza mediante el derecho que HART menciona en otros lugares son la detención de grupos enemigos en tiempo de guerra, de enfermos mentales y la cuarentena (PaR: 17). En LLM: 55-56, HART distingue cuatro modos de hacer uso de la fuerza a fin de hacer valer el derecho — cuatro modos de «legal enforcement of any kind of conduct»: (1) «coertion», o sea, «securing, by the threat of legal punishment, that people do or abstain from doing what the law enjoins or forbids»; (2) «the actual punishment of those who have broken the law»; (3) «steps may be taken which render disobedience to the law impossible or difficult, and so frustrate it rather than punish it», (4) «the use of pressure to induce those actually engaged in breaking the law or threatening to do so to desist»-—. Éste habría sido un buen 


\subsection{El derecho como vehículo de poderes sociales}

¿Pero por qué se trataría de una limitación? ¿En qué sentido el derecho refleja, sanciona, corrobora, constituye relaciones de poder social más allá de la coerción (y que no son poderes normativos)? ¿Qué otras relaciones?

Se dirá: que el derecho sirva al poder es cuestión que se refiere a su contenido contingente. Si, por ejemplo, el derecho sirve a la hegemonía burguesa, esto se refiere a su particular contenido en un particular contexto histórico social y no es, por tanto, materia de investigación de una general jurisprudence.

Esto es cierto. Pero las cosas no son tan simples. Hay otras maneras en las que el derecho «sirve al poder», otros tipos de poder a los que sirve que no son liquidables como una cuestión de contenidos históricos contingentes. Se enumeran ahora algunas ${ }^{14}$.

Primera. Es, sin embargo, oportuna una precisión. En cuanto sigue confiaré en una comprensión intuitiva de la noción de poder que no analizaré. Naturalmente, se trata de una noción problemática. Según algunos autores (LUKES, 1974), se trataría de una essentially contested concept. El término «poder» (poder social) es polisémico. Hay análisis de la noción de poder que establecen condiciones muy restrictivas para la aplicación del término (por ejemplo, que «poder» en la acepción relevante, designa siempre y únicamente una relación entre dos individuos identificables) —análisis a la medida de los cuales la «variedad de poderes» identificados y discutidos en las páginas que siguen (o al menos algunas de ellas) no son en absoluto formas de poder-. Un desarrollo incluido en mi argumentación requeriría una redefinición explicativa, adecuada a la noción. No me empeñaré aquí sobre este tema. Como he dicho, confiaré en una noción intuitiva, seguramente vaga, imprecisa, difuminada de poder y de poder social. Pero espero que la legitimidad del uso de esta noción y de la cualificación de los fenómenos que señalaré como variedad del «poder» resulten evidentes.

(1) El anti-Rule of Law). Una primera vía a recorrer para descubrir en qué otros modos puede suceder que el derecho «sirva» — sea el vehículo— al poder social consiste en examinar qué formas puede asumir aquél en el caso de que sean sistemáticamente violados o no satisfechos los requisitos del Rule of Law. Me explico.

Con la expresión «Rule of Law» entiendo, adoptando la acepción devenida corriente en gran parte de la teoría del derecho de los últimos cuarenta años ${ }^{15}$, un conjunto de (1) propiedades formales de las normas jurídicas (irretroactividad, publicidad, relativa

inicio (un inicio magistral, naturalmente) para una tipología de las variedades del derecho como poder. Pero la teoría del derecho desarrollada en CL queda, bajo este aspecto, incompleta.

${ }^{14} \mathrm{La}$ falta en CL de un tratamiento del «modo en que las formas de derecho interactúan con el poder social» había sido destacada por L. GREEN en un ensayo escrito con ocasión de la publicación de la segunda edición del libro (GREEN, 1996: 1704). GREEN hacía aquí referencia expresa a los modos en que el derecho «incorpora y ejercita» (ibid.) formas de «poder social» diferentes de la coerción e indicaba las discusiones que se siguen abajo (3), (4) y (6).

15 Las concepciones pertenecientes a esta familia tienen la forma de «a sort of laundry list of features that a healthy legal system should have. These are mostly variations of the eight desiderata of Lon Fuller's "internal morality of law"» (WALDRON, 2002: 154). Cfr. ibid., 154-155, para una reseña de las principales concepciones de este tipo (L. L. FulLER, J. RAZ, J. FinNIS, J. RAWLS y M. RADIN; a la que ahora se añade la concepción presentada en KRAMER, 2007: Cap. 2). 
generalidad, relativa estabilidad, inteligibilidad y relativa claridad, praticabilidad ${ }^{16}$ y coherencia) más (2) algunos desiderata procedimentales e institucionales (como, por ejemplo, que la producción de normas individuales que se aplican a casos individuales, sea guiado por reglas generales y, por demás, los denominados «principios de justicia natural»: que la resolución de controversias corresponda a un sujeto no interesado cuyo juicio no esté, por otro lado, viciado; el principio audi alteram partem, etc. $)^{17}$.

Según HART, como es conocido, la conformidad a los requisitos del Rule of Law (en adelante, por la brevedad, RoL) no está en línea de plena constitutividad del concepto de derecho. O sea, puede ocurrir que un ordenamiento social viole sistemáticamente los requisitos del RoL (¿Hasta qué punto? Ésta es una cuestión controvertida no relevante a nuestros fines) ${ }^{18}$ y sea, todavía, derecho (merezca plenamente la calificación de «derecho»). No obstante, HART no indaga en las peculiares relaciones de poder que pueden instaurarse - o consolidarse, encontrar expresión y confirmación, etc. - cuando un método de control social opera mediante la violación sistemática (continua, radical, metódica) de, al menos, algunos de estos requisitos. Así, por ejemplo, la violación sistemática del requisito de la coherencia de las prescripciones jurídicas, o el relativo a que puedan ser cumplidas (el principio «deber» implica «poder») puede generar regímenes de poder caracterizados por el terror, el castigo indiscriminado de los sujetos, un profundo sentido de desconcierto de parte de estos últimos (en el límite la pérdida del sentido de dignidad o de propia autoestima), una radical incerteza y la imposibilidad de planificar las propias acciones y el curso de la propia vida (CELANO, 2011a). Un derecho cuyo contenido no sea de dominio público podrá tener consecuencias análogas (CELANO, 2011b) y podrá, de la misma manera, constituir el vehículo de una forma peculiar de poder social.

Lo mismo vale, en distinto modo y medida, para gran parte de otros desiderata que constituyen el RoL. El principio general es: si quiere ver la variedad de modos en que el derecho puede «servir» al poder el (reflejar, confirmar, consolidar, generar formas de) poder social cuestiona cuál sería la situación si el requisito $\mathrm{X}$ (uno de los requisitos constitutivos del RoL) quedase sistemáticamente incumplido y qué formas de poder podrían prosperar gracias a este incumplimiento sistemático. Como ha puesto de relieve J. RAZ, la violación del RoL, puede provocar un tipo de entrapment (RAZ, 1977, 222); «atrapar» de este modo a las personas es una forma de ejercicio de poder, diferente del ejercicio de la coacción ${ }^{19}$.

16 O sea, conformidad con el principio «deber» implica «poder».

17 Para una lista de estos requisitos institucionales y procedimentales, cfr. por ejemplo, RAZ, 1977: 215 258 [«the making of particular laws (particular legal others) should be guided by open, stable, clear, and general rules»; «the Independence of the judiciary must be guaranteed»; «the courts should have review powers over the implementation for the other principles»; «the courts should be easily accessible»; «the discretion of the crime-preventing agencies should not be allowed to pervert the law»]. Sobre los principios de derecho natural, cfr. HART, 1961: 156; 202; CD: 200, 255; EJP: 115. Para listados análogos de requisitos del Rule of Law, cfr. Fuller, 1969, Capítulo 2; Finnis, 1980: 270-271; MARMOR, 2004: 5 y ss.; KRAMER, 2007: Capítulo 2. Para la subdivisión de los principios del Rule of Law en formales y procedimentales, vid. WALDRON, 2008a (pero $c f r$. ya RAZ, 1977: 218).

${ }^{18}$ Cfr. WALDRON, 2008a. La posición de HarT sobre el punto es oscilante y no del todo transparente; los lugares relevantes son CL: 202; CD: 256; Introduction A EJP: 8-9, 18; L. L. FulLER, The Morality of Law (1965), en EJP: 349-352; EJP: 81, 114-115.

19 Asimismo el RoL es, desde luego, una forma de poder que presenta características peculiares: poder no manipulativo, no paternalista, público y dirigido a sujetos racionales (CELANO, 2011a). 
Pero HART está ciego ante estas diferentes posibilidades. Un derecho que sistemáticamente viole los requisitos del RoL puede convertir a quienes están sometidos a él en objetos de manipulación ${ }^{20} \mathrm{o}$, de cualquier forma, puede conducir a un forma de managerial governance o de «tratamiento» ${ }^{21}$ — que es algo bien distinto de la situación del bandido- («quiero que tú hagas esto y esto otro; estas serán las consecuencias - te causaré este mal - en el caso de que no lo hagas; ahora eres a ti a quien le toca decidir»). HART, naturalmente, tiene bien clara la diferencia; así lo evidencia su análisis (PaR, 23-24; 51-52, 207-208, 232; cfr. de MACCormicK 2008, Cap. XII) de las propuestas de sustitución de la pena con «técnicas manipulativas» (condicionamiento, propaganda, etc.), o con medidas de «higiene social» ${ }^{22}$. Pero de todo esto no hay rastro en CL.

(2) Ideología. Hay un modo peculiar en el que el derecho, típicamente, «sirve» al poder (constituye relaciones de poder social más allá del ejercicio de poder coercitivo) que, en realidad, es bien conocido por HART y que puede también ser reconducido, en parte, a la violación sistemática de algunos requisitos del RoL. Se trata de la bestia negra de BENTHAM: el poder-conocimiento de «Judge E Co.»y, más en general, del «halo de misterio» que las instituciones jurídicas tienden a producir en torno a sí mismas que oscurece «su verdadera naturaleza y efectos», «desconcierta e intimida» al crítico y al reformador (EB, Cap. 1, The Demystification of the Law, en especial: 21). Los elementos constitutivos de esta «mistificación» son (ibid., 21) la «glorificación mediante la oratoria ampulosa», pompa, ceremonia, «el uso de indumentaria arcaica y de fórmulas anticuadas ininteligibles a los legos», y la propagación de una creencia: la creencia en la infinita complejidad y, por tanto, en la inmodificabilidad — sin que ello genere el colapso de la sociedad - de instituciones por largo tiempo estables, asegurando que la actitud adecuada respecto de ellas, aun cuando parezcan ininteligibles, injustas o perjudiciales, sería una postura de deferencia con la tradición.

Este halo de misterio deriva, en parte, de la falta de claridad de las leyes y de su carácter no público. El derecho se presenta como algo esotérico, para iniciados, posesión exclusiva, celosamente guardada, de una casta (sacerdotium juris) y, desde esta perspectiva, es fácil que parezca algo no humano (la criatura se presenta a su creador como una potencia independiente).

En breve, por usar un léxico que en su momento era habitual (sugestivo pero que requiere de análisis), el derecho está empapado de ideología ${ }^{23}$. HART subraya que la consideración de este aspecto del derecho es uno de los elementos centrales de la teoría

20 Fuller, 1969: 38; RaZ, 1977: 331; Finnis, 1980: 273; Kramer, 2007: 184; Celano, 2010: 6, y para un tratamiento sistemático de este punto, 2011a.

${ }^{21}$ FulLer, 1969: 163-167; WALDRON, 2008b: 16-17.

22 Subrayo, para evitar dudas, que lo que está en cuestión no es la diferencia entre «derecho» y «no derecho» (el punto no es que la sustitución de la pena con medidas de higiene social conduciría fuera del campo de aplicación del concepto de derecho; los argumentos de HART contra esta orientación de política criminal no son de este tipo - y, en general, como se ha dicho, la conformidad con los principios del RoL no es para HART, en principio, constitutiva del concepto de derecho-), sino la diferencia entre la situación del bandido (la amenaza con consecuencias desagradables a fin de obtener un cierto comportamiento) y otras formas de poder.

23 Sobre la «rather surprising affinity between Bentham and Karl Marx», cfr. EB: 24 y ss.; ibid., 1-2. Una precisión: hablando de «ideología» no hago referencia aquí al hecho innegable de que las elecciones de política jurídica sean expresión de creencias particulares, preferencias, valoraciones, expectativas, etc. Me refiero, más bien, como se señala en el texto, a un complejo de efectos de poder-saber que surgen del ocultamiento de la na- 
general de derecho de BENTHAM (EB: 22-28). Pero, en CL no se ve ningún rastro ${ }^{24}$. Sin embargo, no se puede sostener que este género de poder (llamémoslo, sólo por disponer de una etiqueta «poder ideológico») esté ligado al derecho de modo accidental ${ }^{25}$.

(3) La selección de los deseos. Consideremos las reglas que confieren poderes a los particulares. Estas reglas ofrecen a los individuos instrumentos, medios idóneos para perseguir, «en el cuadro coercitivo del derecho» (CL: 27; CD: 35), fines, metas, objetivos que ellos prefiguran mediante la creación de estructuras de posiciones jurídicas subjetivas. Pues bien, suministrando a los individuos los instrumentos para perseguir ciertos fines y no otros, el derecho modela - o contribuye a modelar- el abanico de las opciones disponibles para cada uno (por ejemplo, el matrimonio no está disponible a los homosexuales ${ }^{26}$; son posibles los testamentos ordinarios pero no el denominado testamento biológico $)^{27}$ y esto es una forma de poder.

En otros términos, las reglas que confieren poderes (a los particulares) son reglas constitutivas. Qué cosas sean constituidas y qué cosas no — cuáles tipos de acciones y resultados sean puestos a disposición de los individuos y cuáles no- es una cuestión de poder social.

Todavía en otros términos, las reglas que confieren poderes a los particulares «indicate what people should do give up to the wibses they have» (《indican aquello que la gente debe hacer para dar efecto a los propios deseos», CL: 9; CD: 11; cfr. también CL: 27; CD: 34-35, PaR: 45; EJP: 60-61). ¿Qué deseos exactamente? No prever —y, por tanto, impedir - ciertas posibilidades (por ejemplo, el matrimonio homosexual o

turaleza real y de los efectivos mecanismos de funcionamiento, de una particular institución humana (derecho, dinero, bienes ). La noción estaría, por supuesto, terminada.

${ }^{24}$ Una posible alusión: cuando identificación y conocimiento del derecho devienen patrimonio exclusivo de los funcionarios puede ocurrir que todos los demás, como borregos, sean conducidos al matadero (CL: 114; CD: 142-143). Pero ésta es una consideración de carácter más general (vid. la discusión de este punto en el parágrafo 3 infra).

${ }^{25}$ Se piensa, por ejemplo, en el tipo de teoría del derecho practicada por Giovanni TARELLO. Sin embargo, se objetará que no se trata de un nexo conceptual, lógico, entre derecho y poder ideológico. Tampoco, al parecer, es una necesidad (de otro tipo) que el derecho sea un vehículo de poder ideológico. Así, si la teoría del derecho tiene como objeto propio los rasgos necesarios del derecho, una teoría general del derecho podrá, y deberá, investigar como poco los nexos entre derecho y poder ideológico. Este argumento, sin embargo, no es convincente. Vale la pena recordar que, bajo este aspecto, el nexo entre derecho y poder coercitivo no se encuentra en una posición mejor. En la teoría del derecho de los últimos cuarenta años, han sido ideados muchos argumentos ingeniosos a fin de demostrar que la coerción no es un elemento esencial del derecho. De lo que sigue, si la teoría del derecho tiene por objeto los rasgos necesarios del derecho, que el nexo entre derecho y poder coercitivo no deba ser objeto de una teoría general del derecho. Es mucho más plausible, sostengo, la idea de que una investigación teórico-general pueda, y deba, ocuparse también de los rasgos que, incluso no siendo necesarios, a lo menos, típicamente o normalmente (dadas las circunstancias generales de la vida humana) pertenecen al derecho ( $c f r$. SCHAUER, 2010); los dos fenómenos que, tentativamente, SCHAUER aduce como ilustración de su tesis son, precisamente, la coerción y el precedente. Si se acepta esta tesis, el nexo entre derecho y poder ideológico deberá volver a entrar en el campo de investigación de una teoría general del derecho. El nexo subsiste normalmente; el derecho tiene la disposición de servir como vehículo de poder ideológico. Esto es lo que hace de esta relación uno de los objetos propios de una teoría general del derecho (sobre el nexo que según HART subsiste entre derecho y coerción no es ni necesidad conceptual ni dato de facto contingente —cfr. CL: 195; CD: 241-).

26 El ejemplo es utilizado ya en GREEN, 1996: 1703-1704.

27 Sobre los modos en los que el derecho influye —o crea- en las opciones disponibles para la elección (una particular modalidad de incidencia sobre la autonomía individual, diferente del ejercicio del poder coercitivo) $c f r$ RAZ, 1986: 417 y ss. Estoy en deuda con Francesco ZANETTI por algunas sugerencias útiles sobre este punto. 
un testamento dotado de un cierto contenido) es, o, en cualquier caso, puede ser el resultado del ejercicio del poder ${ }^{28}$.

(4) Valor expresivo de la pena. Muchos sostienen que la pena tiene un valor «expresivo» de reprobación y reproche de cara al reo por parte «de la sociedad» (ésta, naturalmente, es una personificación que sería deconstruida) y que la pena «etiqueta» a aquél a quien se la infringe; la institución de la pena sería un mecanismo de clasificación, de construcción social de la identidad. Éstos son, evidentemente, efectos del poder.

HART discute, y critica, la teoría expresiva o etiquetadora del castigo criminal «expressive or denunciatory theory of criminal punishment» (PaR: 2, 7-8, 169 y ss., 235; LLM: 63-65, 83). Sin embargo, lo que él critica es la versión normativa de la teoría según la cual la expresión del reproche o reprobación social es lo que justifica la institución de la pena. A nuestros fines, por el contrario, lo relevante es la versión descriptiva de la teoría según la cual la pena de hecho tiene, típicamente, valor expresivo y desarrolla una función de «etiquetamiento», clasificación, delimitación de una identidad. HART no toma en consideración esta teoría descriptiva que identifica una posterior variedad del derecho como poder social ${ }^{29}$. El derecho penal «etiqueta» y esto es un modo de ejercitar el poder ${ }^{30}$.

(5) Función promocional del derecho. Según algunos, en gran parte de los Estados contemporáneos, el derecho cumple no solamente su tradicional función de imposición de ciertos comportamientos (comisivos u omisivos), mediante la provisión de sanciones negativas (medidas de coerción) sino también la función de promover (pero sin imponer) la adopción, por parte de los ciudadanos, de ciertas líneas de conducta

${ }^{28}$ En LLM: 41.42, HART discute el caso de la pena por bigamia incluso en ausencia de engaño para la otra parte. Concede la nuissance que la bigamia puede constituir para los sentimientos religiosos de la gran mayoría de la población puede ser una justificación suficiente de su punibilidad. Pero, prosigue, en una época de general debilitamiento del sentimiento religioso, la simple invalidez del matrimonio del bígamo podría ser suficiente. Aquí HART roza la cuestión del poder ínsito en la selección de los deseos admisibles ( $c f r$. también EJP: 258-259: «Monogamous marriage is at the heart of our conception of family life, and with the aid of the law it has become part of the structure of society $)$. Es oportuna una precisión. Cuanto se ha dicho en el texto se basa sobre el presupuesto de que efectivamente, como sostiene HART (argumentando a partir de la distinción entre nulidad y sanción), las reglas que imponen obligaciones sean reglas de otro tipo diferente de las reglas que confieren poderes. Como se ha señalado, la tesis de que haya una diferencia de estructura entre estas dos clases de reglas puede ser contestada. En particular, se puede sostener que la diferencia se reduce a esto: las reglas que imponen obligaciones son reglas que comprenden amenazas, las que confieren poderes son offerte de beneficios (así SHAPIRO, 2011: 63-66, rediseña la distinción entre nulidad y sanción, replicando a una — hipotética — replica austiniana a la objeción de HART). $\mathrm{Si}$ las reglas que confieren poderes son comprendidas de este modo, la selección de los deseos será el efecto del hecho de que el derecho formula sólo ciertas ofertas y no otras. $\mathrm{O}$, en otros términos, la forma de poder que he denominado «selección de deseos» recaerá en el ámbito de la «función promocional» del derecho [punto (5) infra].

${ }^{29}$ In PaR: 263 (pero cfr. también ibid., 239; LLM: 63-65, 83) HART distingue entre la versión normativa y la versión definitoria (J. FEINBERG) de la teoría. Pero el punto no es desarrollado (nótese, sin embargo, que HART acoge explícitamente la tesis según la cual un ilícito es diferente porque es el objeto de condena de la conducta el que está sujeto a evaluación; EJP: 301). Este límite de la jurisprudence hartiana había sido ya destacado por MACCORMICK (2008: Cap. XII, nn. 5 y 12).

${ }^{30}$ Las formas en las que el derecho juega un papel en la constitución de la identidad social (roles, status) y, de esta manera, opera como vehículo de poder social, naturalmente, no se explican por el etiquetamiento de aquellos a quienes le es infringida una pena. Piénsese, por ejemplo, en la atribución de capacidad jurídica. Estas formas adicionales son reconducibles, al menos en parte, a la selección de los deseos [supra, sub (3)] y al ejercicio de una función promocional [infra, sub (5)] pero no son, probablemente, enteramente reconducibles a estos dos fenómenos. 
mediante la previsión de sanciones positivas (recompensas) para aquellos que adopten tales comportamientos. Esta función será denominada «función promocional» (BoBBIO, 1977).

Más precisamente, con la expresión «función promocional del derecho» se entiende «la acción que el derecho desarrolla a través del instrumento de las "sanciones positivas”, esto es, a través mecanismos genéricamente comprendidos con el nombre de "incentivos" que se dirigen antes que a impedir actos socialmente indeseables, lo que es el fin principal de penas, multas, correctivos, restituciones, resarcimientos, etc. a "promover" el cumplimiento de actos socialmente deseables» (BOBBIO, 1977: 7).

La tesis de que el derecho cumple en gran parte de los Estados contemporáneos, a diferencia de su tradicional función protectivo-represiva, también una función promocional (esto es, una función de dirección o de orientación de algunas esferas de actividad) está conectada a la distinción entre dos modelos de Estado: el Estado liberal y el Estado social. Simplificando al extremo, el Estado social, a diferencia del Estado liberal, buscar orientar en determinadas direcciones (esto es, impulsar hacia objetivos prefijados) el desarrollo social, conformando una orientación pragmática las actividades sociales relevantes (en particular, la actividad económica; en este sentido, el Estado social contemporáneo ha estado frecuentemente calificado como Estado «dirigista») ${ }^{31}$.

La tesis de que el derecho desarrolla, en el Estado social, una función promocional ha sido objeto de un intenso debate en el que no entraré aquí. Pero no hay duda, en mi opinión, de que esta actividad de dirección u orientación de algunas esferas de actividad social — si es efectiva - constituye una forma de poder $^{32} \mathrm{y}$, por demás, la individualización de un criterio que permita trazar una precisa línea de demarcación entre la amenaza de consecuencias desagradables y la oferta de recompensas es uno de los nudos irresueltos de la teoría de la coerción ( $c f$ r. ANDERSON, 2006, s. 2.4) $)^{33}$.

(6) Poder disciplinario, biopolítica. Si se pudiese dar a las nociones foucaultianas de poder disciplinario y de biopolítica un contenido claro y determinado y si, como parece a primera vista plausible, estas nociones identifican un conjunto de modos, formas, técnicas, relaciones peculiares de poder diferentes del ejercicio de la coerción, la relevancia de estos fenómenos para una teoría general del derecho sería innegable. El derecho está típicamente implicado en la estructuración de estas (hipotéticas) formas y relaciones de poder.

31 El tratamiento de la función promocional del derecho, sostenía BoBBIO (1977: 7), es necesaria «para adecuar la teoría general del derecho a las transformaciones de la sociedad contemporánea y al crecimiento del Estado social o prestacional o de bienestar o de justicia o de capitalismo monopolístico, como de forma más o menos benevolente se quiera llamar» (una «necesaria adecuación» para «comprender el paso del Estado "garantista" al Estado "dirigista", y consecuentemente la metamorfosis del derecho de un instrumento de "control social", en el sentido estricto del término, en instrumento de "dirección social"».

32 Conste que no es necesariamente un rasgo que sería propio, en sentido estricto, del Estado social del siglo XX. La función promocional del derecho, observa BOBBIO, «no es nueva pero sí es novedosa la extensión que ha tenido y continúa teniendo en el Estado contemporáneo» (BOBBIO, 1977: 8).

33 HART, en obras posteriores a CL (EB: 109, 118, 123, 139, 201), suprime la referencia, en la jurisprudence benthamita, a la categoría de «leyes de recompensa»; leyes que, precisamente, se orientan a dirigir el comportamiento de aquellos que se someten mediante la oferta de ventajas. Pero se trata solamente de alusiones no desarrolladas. En conjunto, parece que HART comparte la opinión de BENTHAM (rectius, la opinión que el propio HART atribuye a BENTHAM) de que las leyes de este tipo no pueden ser más que raras o, en todo caso, de importancia secundaria. 


\section{DERECHO Y PODER POLÍTICO}

¿Qué hay de la relación entre derecho y poder político en el CL?

HART, como es sabido, sostiene una serie de objeciones en relación con la noción austiniana del soberano como fuente, directa o indirecta, de todo el derecho. La teoría benthamita y austiniana del soberano era el fruto, vía Hume (sobre el contrato originario; Hume, 1748), del rechazo de las teorías del contrato social y otras ficciones similares $^{34}$. El «fundamento» (uso calculadamente este término ambiguo entre explicación y justificación: la ambigüedad está precisamente en las teorías que BENTHAM y AuSTIN rechazan) del poder político no está en un imaginario pacto entre individuos libres e iguales en un estado de naturaleza. El poder político es ante todo un hecho: el hecho del dominio y el hecho de la aquiescencia, de la obediencia habitual.

HART rechaza, por razones conceptuales (cuya contundencia no pongo en cuestión), esta teoría. Pero ¿qué propone en su lugar?

HART sostiene que en la base de la autoridad de cualquier gobernante (se trate del imaginario Rex o del King in Parliament) hay, por necesidad conceptual, una regla social: la aceptación compartida por parte de los miembros del grupo de un cierto pattern regular de comportamiento como un standard al que atenerse (CL: 56-57; CD: 7072). Esta regla es una regla consuetudinaria que HART llama «regla de reconocimiento» (que, necesariamente, se refiere a los resultados de la actividad definida por las reglas de cambio; CL: 93; CD: 117-118). Sin embargo, para que exista un sistema jurídico no es necesario que esta costumbre —el uso de ciertos criterios a fin de la identificación del derecho válido - sea difusamente sostenida por todos los miembros del grupo. Basta que lo sea entre los funcionarios, ante todo entre los jueces; en un Estado moderno es suficiente que la mayoría tenga un comportamiento de aquiescencia (CL: 59-60, 113; CD: 75, 140).

¿Cuál es, entonces, la imagen que CL expone del fundamento de un sistema jurídico? (foundations: es el término de HART —CL, Cap. VI, The Foundations of a Legal System-; no advierte aquí, con precisión, en qué sentido lo utiliza, si sólo explicativo o también normativo). Para responder a esta pregunta es necesario distinguir entre la primera edición del libro (1961) y el Postscript (publicado en 1994).

En 1961, el fundamento de un sistema jurídico es, como ya se ha esbozado, una práctica social de los funcionarios — ante todo los jueces - consistente en hacer uso de ciertos criterios a los fines de identificación del derecho válido. Sería una costumbre, como aquélla según la cual los hombres deben quitarse el sombrero al entrar en una iglesia. Pero esto es una imagen un tanto extraña, reductiva, del complejo de hechos sobre los cuales reposa la existencia de un sistema jurídico, una imagen que rescinde los vínculos entre el hecho de la existencia de un sistema jurídico y los conflictos, las luchas, las agitaciones, las relaciones de poder, en los cuales tiene su origen y en cuyo contexto se desarrolla. Una imagen que se podría calificar de «quietista» como si la existencia y el funcionamiento del derecho fuesen sólo cuestión del des-

34 Extremos, obviamente, bien conocidos por HART (EB: 221). Entre la teoría de la soberanía de BENTHAM y la de AUSTIN hay profundas diferencias analizadas y puestas de relieve por HART (EB: 108-109; 225-227; cfr. también EJP: 55), pero que son, a nuestros efectos, irrelevantes. 
envolvimiento ordinario de la actividad de disciplinados operadores jurídicos, sobre todo, los jueces ${ }^{35}$.

En el Postcript, la respuesta viene más precisada. El fundamento de un sistema jurídico, la regla de reconocimiento, es una convención. Una convención es una regla social de un tipo particular: cada uno de los miembros del grupo relevante (en el caso de la regla de reconocimiento, cada funcionario) se comporta en un cierto modo (hace uso de ciertos criterios de identificación del derecho válido) porque, inter alia, se espera que los otros miembros del grupo (los otros funcionarios) se comportarán en esa determinada manera (porque cada uno de ellos espera que los otros miembros del grupo se comporten en esa manera y así sucesivamente) ${ }^{36}$. Se trata de un fenómeno análogo al que ocurre con ciertos locales de moda: cada uno va porque espera encontrarse con los otros porque éstos esperan encontrarse con los anteriores, y así sucesivamente. La existencia de un sistema jurídico es un fenómeno asimilable, en última instancia, al hecho de que todos se vayan al pub el sábado por la noche. Esto siempre limitadamente al club de los funcionarios.

Esta respuesta del Postcript es, como puede verse, una imagen del fundamento de un sistema jurídico todavía más quietista (en el sentido precisado) ${ }^{37}$.

La impresión es confirmada si se mira la génesis ideal de un sistema jurídico que HART presenta al final del Capítulo 5 del CL (89 y ss. CD: 116 y ss.) y al tratamiento del derecho natural mínimo (CL: 189-195; CD: 237-243; cfr. también EJP: 79-80).

(1) La génesis ideal de un sistema jurídico desarrollado.

Un régimen que sólo tenga reglas sociales que impongan obligaciones, argumenta HART, presentaría tres defectos: incerteza sobre cuáles serían las reglas de grupo; imposibilidad de modificar deliberadamente estas reglas; ineficiencia de la presión social en relación a quien viola o amenaza violar las reglas. A estas tres deficiencias pone remedio la introducción de reglas de segundo nivel (reglas sobre reglas): respectivamente, reglas de reconocimiento, reglas de cambio y reglas de adjudicación. Con la introducción de estas reglas, concluye HART, vemos nacer ante nosotros un sistema jurídico desarrollado, unión de reglas primarias y secundarias.

¿Qué género de reconstrucción es ésta? Las deficiencias características de un régimen que exclusivamente tenga reglas sociales de obligación parecería que son percibidas claramente. ¿Por quién? HART parece imaginar una población de individuos generalmente bienintencionados (pero que tampoco son ángeles) que buscan conjuntamente el modo de evitar las dificultades comúnmente advertidas: hacer eficiente la «propia» presión sobre los recalcitrantes; hacer menos inciertas sus reglas; hacer frente, asimismo, a las exigencias de modificación de las reglas. Individuos más o menos iguales que están sobre un plano de paridad y buscan los medios idóneos para remediar los defectos de que adolece su régimen de convivencia es una representación, en su conjunto, carente de realismo y edulcorada.

35 Es cierto que HART puntualiza que la aceptación de la regla de reconocimiento puede tener las más diversas razones (CL: 112-113; CD: 140-141) pero esto no colma la laguna.

36 Para un tratamiento detallado $c f r$. CELANO, 2003.

37 HART aclara que donde sólo son los funcionarios lo que aceptan la regla de reconocimiento y hacen uso de ella, los restantes son como borregos y los borregos pueden terminar en el matadero [CL, 114; CD: 145; J. WALDRON (1999) ha subrayado justamente este paso de CL]. Pero esto es realmente poco. 
Pero se objetará que esto es forzado. HART no presenta la propia construcción como la narración de un proceso histórico. Es cierto. Pero el problema no es aquello que hay en el análisis de HART sino aquello que falta. Lo que hay, la reconstrucción de la génesis ideal de un sistema jurídico a partir de un régimen exclusivamente formado por reglas sociales de obligación, sugiere precisamente la imagen irreal y edulcorada que se ha dicho. Vayamos de frente, parecería una enésima versión de la salida del estado de naturaleza. La población cuyo ingreso en el mundo jurídico imagina está representada, implícitamente, como un grupo de individuos aproximativamente iguales, ni diablos ni ángeles, grosso modo racionales y que se encuentra, grosso modo, sobre un plano de paridad. Ésta es la dotación del derecho natural mínimo.

(2) El contenido mínimo del derecho natural ${ }^{38}$.

HART demuestra, o pretende demostrar, que, por una necesidad natural, en cualquier grupo social (que no sea un club de suicidas) existe un cierto conjunto de reglas que se dirigen fundamentalmente a la evitación de actos de violencia arbitraria y otros similares.

La demostración presupone, sin embargo, una cierta representación de las disposiciones de poder en la sociedad. O mejor, una representación de la sociedad como no —o no todavía - marcada por relaciones de poder: el estado de naturaleza.

El derecho natural mínimo es presentado por HART como un conjunto de reglas aceptadas y generalmente observadas por un grupo de individuos (limitadamente) racionales y (limitadamente) bien dispuestos, aproximadamente iguales y que no están en relaciones de poder los unos con los otros; que, como individuos, se confrontan sobre un plano de igualdad. Ésta es, repito, la enésima versión de la idea del estado de naturaleza. El argumento que sostiene la tesis del contenido mínimo del derecho natural no presupone solamente los bien conocidos «axiomas» acerca de la naturaleza humana y las circunstancias en las que viven los hombres que HART explícitamente menciona; presupone también una particular representación de la disposición de las relaciones sociales, la imagen de individuos entre los cuales no existen todavía relaciones de poder ${ }^{39}$.

Es cierto (era esta pregunta, como se recordará, la segunda objeción contra mi tesis; supra, 2): HART afirma y confirma repetidas veces que puede ocurrir que un sistema jurídico reserve su protección —en particular la protección que deriva del conjunto de «abstenciones recíprocas» que constituye el núcleo de su contenido natural mínimoy demás beneficios que derivan de su existencia, a una clase particular de individuos a

38 Para una reconstrucción minuciosa de la doctrina hartiana del contenido mínimo del derecho natural, de sus presupuestos teóricos, del contexto histórico-filosófico en el que se desarrolla y de su papel en la teoría del derecho de HART, $c f r$. RICCIARDI, 2008, en particular, 234 y ss.

39 Por dos veces, discutiendo de la igualdad aproximada y de un sistema de sanciones socialmente organizado (CL: 194; CD: 247), HART menciona la escena internacional como un ámbito caracterizado por un diferente equilibrio de base: un diferente equilibrio de poder («conflicting power centers» de diferente peso) en el cual, precisamente en razón del diferente equilibrio de las relaciones de poder, no existe un derecho natural (mínimo). Esto significa que la teoría del derecho natural mínimo se basa sobre una cuestión acerca del equilibrio «de partida» del poder en las sociedades estatales: no uno de los axiomas enumerados por HART sino uno de los asuntos empíricos no explicitados (y menos aún argumentados) y poco realistas: la idea de un estado de naturaleza en el que los miembros del grupo no están (todavía) inscritos en relaciones de poder ( $c f r$., en el mismo sentido, CL: 214-5; CD: 271: «Municipal law, in its setting of physical and psychological facts», un setting diferente del que corresponde al derecho internacional, «[a] very different background of fact»). 
los que se aplican sus reglas (una «ruling élite», CL: 195-8; CD: 247-250, cfr. también CL: 161, 175; CD: 207-208, 224; EJP: 81, 116), o que les reserva una protección mayor o beneficios mayores respecto a los otros miembros del grupo. Allí donde existe un sistema jurídico puede ocurrir que sean «slave or subject clases» (CL: 201; CD: 254). Dice HART, a las claras: «So long as buman beings can gain sufficient co-operation from some to enable them to dominate others, they will use the forms of law as one of their instruments» («mientras que los seres humanos puedan obtener suficiente cooperación de algunos para permitirles dominar a otro, usarán las formas del derecho como uno de sus instrumentos», CL: 205-206; CD: 259).

Ésta es ciertamente una manera, de crucial importancia, en la que el derecho refleja y concurre a constituir relaciones de poder político. Se trata, efectivamente, de una - significativa - excepción respecto a cuanto se ha dicho hasta ahora. HART no está del todo ciego a las posibles relaciones entre derecho y poder (político). De acuerdo. Pero se trata, precisamente, de sólo una — aunque sea importante- excepción. HART no dice nada más sobre el tema. La posibilidad de que el derecho no extienda su protección a una clase subordinada es sólo uno entre las diferentes maneras en que puede ocurrir que el derecho «sirva» al poder político ${ }^{40}$.

\section{CONCLUSIÓN}

HART — con la importante excepción indicada al final del párrafo precedente — no ve el derecho como el instrumento y sistema de poder: no ve las variedades del poder (utilizando esta noción, es cierto - supra, par. 2- en forma intuitiva y no refinada) con las que el derecho está típicamente (aunque no necesariamente; supra, par. 2) entrelazado. En particular, se desembarazada del modelo austiniano del soberano - que es precisamente un modelo, por más que insatisfactorio, de poder político- pero no lo sustituye con ningún otro (supra, par. 3). Salvo quizá con la imagen poco realista y tendencialmente mistificadora de una comunidad de jueces cohesionada y consistente (pero esto, tal vez, es más un rasgo típico de alguna relaboración post-hartiana de la idea hartiana de que el fundamento de un sistema jurídico está constituido por una práctica concordante de los funcionarios).

Es cierto: (1) HART individualiza en el derecho normas que confieren poderes. Pero se trata de poderes normativos: poderes, instituidos por normas, de producción y aplicación de normas. Aquí se discute de poderes físicos y psicológicos: del derecho como vehículo de poder social. (2) Para HART, el derecho está compuesto también por normas que imponen obligaciones, provistas de la previsión de sanciones punitivas para el caso de incumplimiento. Pero: a) HART no provee ningún análisis de este modo de ejercitar el poder (desde este punto de vista su teoría es aún más «pura» que la de KELSEN que al menos analiza el mecanismo de la motivación indirecta; KELSEN, 1945: 15 y ss.); b) éste es sólo uno de entre los diversos aspectos del derecho como

40 Se puede considerar una segunda excepción lo que dice HART sobre los casos en que jueces de última instancia determinan textura abierta de la regla de reconocimiento; los casos del tipo all that succeeds is success («el poder adquiere autoridad ex post facto de su éxito», CL: 149-150; CD: 190-191). Pero esto concierne, aún otra vez, a lo que sucede en el interior de la comunidad de los jueces. 
poder — sólo una de las variedades del poder social de las cuales el derecho puede ser el vehículo-.

Así, la teoría del derecho resulta anulada por la teoría política y la investigación sobre otras variedades del poder, como si el derecho no tuviese nada que ver con el dominio, el conflicto social, el control (más allá de la coerción o, también, en general, del uso de la fuerza) sobre los individuos. La teoría del derecho deviene una descripción - una descripción interminable en la obra de los epígonos- de las prácticas del club de funcionarios.

(Traducción de José María Sauca)

\section{REFERENCIAS BIBLIOGRÁFICAS}

\section{Obras de HarT}

- 1949: «The Ascription of Responsibility and Rights», en Proceedings of the Aristotelian Society, New Series, 49 (1948-1949).

— 1955: «Are There Any Natural Rights», reed. en J. WALDRON (ed.), Theories of Rights, Oxford: Oxford University Press, 1984.

- 1958: «Legal and Moral Obligation», en A. I. MeLden (ed.), Essays in Moral Philosophy, Seattle: University of Washington Press, 1958.

- 1961: CL, The Concept of Law, Oxford: Clarendon Press. Versión española, El concepto de Derecho, trad. G. R. CARRIÓ, Buenos Aires: Abeledo Perrot, 1977.

- 1963: LLM, Law, Liberty and Morality, Oxford: Oxford University Press.

- 1968: PaR, «Punishment and Responsibility», en Essays in the Philosophy of Law, Oxford: Clarendon Press.

- 1982: EB, Essays on Bentham. Studies in Jurisprudence and Political Theory, Oxford: Clarendon Press.

- 1983: EJP, Essays in Jurisprudence and Philosophy, Oxford: Clarendon Press.

- 1987: Comment, «Comment», en R. GAvison (ed.), Issues in Contemporary Legal Philosophy. The Influence of H. L. A. Hart, Oxford: Clarendon Press.

- 1994: Postscript, «Postscript» a H. L. A. HART, The Concept of Law, 2. ${ }^{a}$ ed., Oxford: Clarendon Press.

\section{Otras obras citadas}

ANDERSON, S., 2008: «Coercion», en E. N. ZALTA (ed.), The Stanford Encyclopedia of Philosophy, (Fall, 2008 Edition), http://plato.stanford.edu/archives/fall2008/entries/coercion/.

BoBBiO, N., 1977: Dalla struttura alla funzione, Milano: Comunità.

Celano, B., 2002: «I diritti nella jurisprudence anglosassone contemporanea. Da Hart a Raz», en P. Comanducci y R. GuASTINI (a cura di), Analisi e diritto. Ricerche di giurisprudenza analitica, Torino: Giappichelli, 2002, 1-58 (bttp://www.giuri.unige.it/intro/dipist/digita/filo/ testi/analisi_2001).

- 2003: «La regola di riconoscimento è una convenzione?», en M. C. REDONDO (a cura di), «Il Postscript di H. L. A. Hart», en Ragion pratica, n. 21,347-360. 
— 2010: «Positivismo jurídico normativo, neutralidad y estado de derecho», en J. FERRER BELTRÁN, J. J. Moreso y D. M. PAPAYANNIS, Neutralidad y teoría del derecho, Madrid: Marcial Pons, 2012, 243-278.

- 2011a: «Law as Power: Two Rule of Law Requirements», en W. WaLuCHOw y S. SCIARAFFA (eds.), Philosophical Foundations of the Nature of Law, New York: Oxford University Press.

- 2011b: «Publicity and the Rule of Law», en L. GrEen y B. LEITER (eds.), Oxford Studies in Philosopby of Law, vol. 2, Oxford: Oxford University Press.

FinNis, J. M., 1980: Natural Law and Natural Rights, Oxford: Clarendon Press.

Fuller, L. L., 1969: The Morality of Law, revised edition, New Haven: Yale University Press.

GreEN, L., 1996: «The Concept of law Revisited», Michigan Law Review, 94, 565-580.

Hume, D., 1748: «Of the Original Contract», en E. BARKer (ed.), Social Contract. Essays by Locke, Hume and Rousseau, Oxford: Oxford University Press, 1947.

Kelsen, H., 1945: General Theory of Law and State, Cambridge (Mass.): Harvard University Press.

Kramer, M. H., 2007: Objectivity and the Rule of Law, Cambridge: Cambridge University Press.

Lukes, S., 1974: Power: A Radical View, London: Macmillan.

MacCormick, N., 2008: H. L. A. Hart, 2. ${ }^{a}$ ed., Stanford (Calif.): Stanford University Press (1. ${ }^{\text {a }}$ ed., 1981).

Marmor, A., 2004: «The Rule of Law and Its Limits», en Law and Philosophy, 23, 1-43.

RAZ, J., 1977: «The Rule of Law and Its Virtue», en J. RAZ, The Authority of Law. Essays on Law and Morality, Oxford: Clarendon Press, 1979.

- 1986: The Morality of Freedom, Oxford: Clarendon Press.

Ricciardi, M., 2008: Diritto e natura. H. L. A. Hart e la filosofia di Oxford, Pisa: Edizioni ETS.

SCHAUER, F., 2010: «Necesidad, importancia y naturaleza del Derecho», en J. FERRER BELTRÁN, J. J. Moreso y D. M. PAPAYANnis, Neutralidad y teoría del derecho, Madrid: Marcial Pons, 2012, 41-59.

SHAPIRO, S. J., 2011: Legality, Cambridge (Mass.): Harvard University Press.

WALDRON, J., 1999: «All We Like Sheep», en Canadian Journal of Law and Jurisprudence, 12, 1, 169-186.

- 2002: «Is the Rule of Law an Essentially Contested Concept (in Florida)?», en Law and Philosophy, 21, 2, 137-164.

- 2008a: «Hart and the Principles of Legality», en M. KrAmER et al. (eds.), The Legacy of H. L. A. Hart. Legal, Political, and Moral Philosophy, Oxford: Oxford University Press, 2008.

— 2008b: «The Concept and the Rule of Law», en Georgia Law Review, 43, 1-61. 